\section{Análise de tendências das taxas de mortalidade infantil e de seus fatores de risco na cidade de Porto Alegre, Rio Grande do Sul, Brasil, no período de 1996 a 2008}

\author{
Analysis of infant mortality trends and risk factors \\ in Porto Alegre, Rio Grande do Sul State, Brazil, \\ 1996-2008
}

Alessandra Rivero Hernandez 1,2 Clécio Homrich da Silva 1,2,3 Marilyn Agranonik 1,2 Fernanda Maciel de Quadros 2 Marcelo Zubaran Goldani 1,2,3

\title{
Introdução
}

1 Programa de Pós-graduação em Saúde da Criança e do Adolescente, Universidade Federal do Rio Grande do Sul, Porto Alegre, Brasil.

2 Núcleo de Estudos de Saúde da Criança e do Adolescente da Universidade Federal do Rio Grande do Sul, Porto Alegre, Brasil.

3 Faculdade de Medicina, Universidade Federal do Rio Grande do Sul, Porto Alegre, Brasil.

Correspondência A. R. Hernandez Programa de Pós-graduação em Saúde da Criança e do Adolescente, Universidade Federal do Rio Grande do Sul. Av. Cristóvão Colombo 3.038, apto. 301, Porto Alegre, RS 90560-002, Brasil.

riverohernandez@hotmail.com

\section{Abstract}

The aim of this article was to determine trends in infant mortality and related risk factors in Porto Alegre, Rio Grande do Sul State, Brazil. The study was based on data from a live birth and infant death registry for the years 1996 to 2008. Times trends were analyzed for overall and partial infant mortality rates according to maternal schooling, number of prenatal visits, maternal age, number of live and dead children, sex of the newborn, type of delivery, birth weight, and type of hospital. Poisson regression was used to estimate the influence of socioeconomic status and other risk factors. The infant mortality rate dropped among mothers with less than 11 years of schooling. There were no significant changes among newborns of mothers with 12 or more years of schooling. Maternal socioeconomic status was the factor most closely associated with the reduction in infant mortality. Still, the downward trend failed to achieve its full potential, due to the increase in low birth weight.

Infant Mortality; Mortality Rate; Social Inequity; Low Birth Weight Infant
No Brasil, nota-se uma diminuição significativa das taxas de mortalidade infantil nas últimas décadas 1. Entretanto, a manutenção de um alto grau de desigualdade social tem sido relacionada com a permanência de taxas de mortalidade infantil ainda elevadas, principalmente nos grupos sociais menos privilegiados 2,3,4,5. Além disso, o baixo peso ao nascer, considerado o fator individual mais importante associado à morbimortalidade infantil 6 , tem aumentado paradoxalmente em algumas regiões do país 7,8, fato que tem sido, muitas vezes, associado ao aumento de nascimentos por cesárea $9,10,11$.

Em geral, os estudos dedicados a avaliar a tendência de mortalidade infantil e seus fatores correlatos consideraram as diferenças sociais e o acesso desigual a diversos aparelhos sociais, tendo, como base, estudos de coorte com intervenções pontuais realizadas em momentos diferentes dentro de intervalos que representam determinados períodos de tempo ou bancos de dados com séries de curta duração, não permitindo determinar, com mais precisão, a tendência secular dessa taxa, bem como o impacto desses fatores ao longo do tempo 2,3,4,5,12. Outra limitação presente em muitos estudos que utilizam bases de dados é analisar apenas as taxas de mortalidade infantil e associá-las a dados agregados, não havendo, dessa forma, informações individuais 
sobre as características maternas, a gestação, o parto e o recém-nascido 3,5,12,13,14,15,16,17.

Portanto, o objetivo deste estudo é contribuir para a análise das tendências das taxas de mortalidade infantil, neonatal e pós-neonatal e de seus fatores de risco, investigando uma longa série temporal na cidade de Porto Alegre, Rio Grande do Sul, Brasil.

\section{Métodos}

Foi realizado um estudo de séries temporais em Porto Alegre, cidade com 1.409.939 habitantes, localizada na Região Sul do Brasil (Instituto Brasileiro de Geografia e Estatística. Censo 2010. http://www.ibge.gov.br/cidadesat/topwindow. htm?, acessado em 15/Dez/2010). Foram coletadas, no Sistema de Informações de Nascidos Vivos (SINASC), as informações sobre todos os nascidos vivos únicos de mães residentes no município nos anos de 1996 a 2008. O SINASC foi implantado no Brasil em 1993, com base na Declaração de Nascido Vivo (DNV), e possui informações relativas às características maternas, à gravidez, ao parto e ao recém-nascido 18 . A partir do SINASC, foram utilizadas as seguintes variáveis: o número de nascidos vivos, a escolaridade materna (menos de 8 anos, de 8-11 anos, 12 ou mais anos), o número de consultas pré-natal (nenhuma consulta, 1-6 consultas, 7 ou mais consultas), a idade materna ( $\leq 17$ anos; 18-20 anos; 21-30 anos; 31-35 anos; > 35 anos), o número de filhos vivos anteriores (nenhum; 1-2 filhos; 3-4 filhos; $\geq 5$ filhos), o número de filhos mortos anteriores (nenhum; $\geq 1$ filhos), a idade gestacional ( $\leq 36$ semanas; $37-41$ semanas; $\geq 42$ semanas), o tipo de nascimento (parto vaginal; parto operatório), o tipo de hospital (público; privado; misto), o peso de nascimento (500g999g; 1.000g-1.499g; 1.500g-2.499g; 2.500g-2 .999g; 3.000g-3.999g; $\geq 4.000 \mathrm{~g}$ ) e o sexo do recém-nascido (feminino; masculino). Propôs-se uma estratificação do nível de desigualdade social pela escolaridade materna.

Foi criada a variável índice ambiental por meio de análise fatorial das seguintes informações dos bairros de Porto Alegre obtidas no censo demográfico de 2000 19: percentuais de domicílios sem banheiro nem sanitário, com abastecimento de água não adequado, com esgotamento sanitário não adequado e com lixo não coletado. Depois, essa variável foi incluída no banco de dados, e cada nascido vivo obteve o valor do índice correspondente ao bairro de residência da mãe, informação essa coletada do SINASC.

A ocorrência e o período de óbito foram coletados no Sistema de Informações sobre Mor- talidade (SIM). O SIM foi criado em 1975, com base nas informações oriundas da Declaração de Óbito (DO) 18. Foram excluídos das análises todos os recém-nascidos com peso ao nascer abaixo de $500 \mathrm{~g}$, pela possibilidade de sobrevivência extremamente baixa, contribuindo, de forma desproporcional, para as taxas de mortalidade infantil 20,21, e os nascimentos múltiplos, por apresentarem um padrão diferenciado de mortalidade infantil e de seus fatores de risco, com maior frequência de prematuridade e de restrição de crescimento fetal 22,23,24,25,26, totalizando 5.920 nascidos vivos excluídos.

Um estudo realizado em 1998 verificou que, no Rio Grande do Sul, 64,3\% da população apresentou informações adequadas de nascimentos e de óbitos, satisfazendo todos os critérios analisados, enquanto apenas $1,4 \%$ da população apresentou um grau acentuado de precariedade dos dados de óbitos 27. Em Porto Alegre, os dados do SINASC e do SIM apresentam uma excelente completude e um percentual baixo de ignorados 28 .

Os bancos de dados foram fornecidos pela Secretaria Municipal de Saúde, no formato de bancos anuais. A partir de então, foram desenvolvidos dois bancos de dados com séries temporais, um para cada sistema de informação. As variáveis do estudo encontram-se categorizadas, e aquelas que, durante o período, sofreram alterações foram padronizadas posteriormente. $\mathrm{O}$ link entre os dois sistemas foi obtido automaticamente por intermédio do número do certificado de nascido vivo, presente nos dois sistemas de informação, em 96,6\% dos óbitos. Quando não houve concordância entre o certificado de óbito e o respectivo de nascimento $(3,4 \%)$, o link foi realizado, manualmente, pelo nome da mãe que, posteriormente, foi confirmado pelo peso e data de nascimento do recém-nascido. O percentual de perdas dos óbitos foi de 3,5\%.

A taxa de mortalidade infantil foi calculada pelo número total de óbitos em menores de um ano dividido pelo número total de nascidos vivos por mil, considerando o ano de nascimento para o número de óbitos e de nascidos vivos. Assim, se a criança nasceu em 2008 e morreu em 2009, antes de completar um ano de vida, ela foi incluída na taxa de mortalidade para o ano de 2008. A mesma lógica foi mantida para estimar a mortalidade neonatal (até 27 dias) e a pós-neonatal (28-364 dias).

A regressão linear foi utilizada para analisar as tendências de nascidos vivos e das taxas de mortalidade, neonatal e pós-neonatal, gerais e de acordo com: a escolaridade materna, o número de consultas de pré-natal, a idade materna, o número de filhos vivos e mortos, o sexo do 
recém-nascido, o tipo de parto, a idade gestacional, o peso de nascimento e o tipo de hospital. $\mathrm{O}$ teste qui-quadrado de tendência foi utilizado para avaliar a possível tendência temporal das taxas.

Foi utilizada regressão sequencial de Poisson para estimar a influência da condição socioeconômica (escolaridade materna), das variáveis assistenciais (número de consultas de pré-natal, tipo de parto e tipo de hospital), das variáveis maternas (idade materna e número de filhos vivos e mortos) e das variáveis do recém-nascido (sexo do recém-nascido, idade gestacional e peso de nascimento) nas tendências das taxas de mortalidade infantil, neonatal e pós-neonatal durante o período. $\mathrm{O}$ ano foi incluído no modelo como uma variável contínua, e o efeito do período foi estimado como uma tendência (linear) anual. Os riscos relativos foram calculados com um intervalo de $95 \%$ de confiança. O nível de significância foi estabelecido em $5 \%$ para todas as análises. $\mathrm{O}$ processamento do banco de dados e as análises foram realizados no programa SPSS, versão 17.0 (SPSS Inc., Chicago, Estados Unidos). O projeto de pesquisa foi aprovado pelo Comitê de Ética e Pesquisa da Secretaria Municipal de Saúde da Prefeitura de Porto Alegre.

\section{Resultados}

Durante o período analisado, conforme pode ser visto na Tabela 1, ocorreram 265.242 nascimentos. Houve uma redução de $23,5 \%$ no número de nascidos vivos. As taxas de mortalidade infantil, neonatal e pós-neonatal decresceram de forma significativa, respectivamente, de 15,8/1.000 nascidos vivos em 1996 para 9,1/1.000 nascidos vivos em 2008 (3,7\% ao ano; valor de $\mathrm{p}<0,001)$, de 8,7 para 5,9 (3,5\% ao ano; valor de $\mathrm{p}<0,001)$ e de 7,1 para $3,0(4,1 \%$ ao ano; valor de $\mathrm{p}<0,001)$. Em oito óbitos, não foi possível identificar o seu período de ocorrência.

Na Tabela 2, observa-se que, em relação aos nascidos vivos, há uma tendência de aumento da escolaridade materna, do número de consultas de pré-natal, da idade materna, da primiparidade, de parto cesáreo, da prematuridade, do baixo peso ao nascer, do muito baixo peso ao nascer e de nascimentos em hospital particular. Houve uma tendência de redução da multiparidade, da idade gestacional igual ou maior a 42 semanas e peso de nascimento igual ou maior a $4.000 \mathrm{~g}$. Quanto à mortalidade infantil, houve uma tendência de redução, considerando as seguintes categorias das variáveis em estudo: menos de 8

Tabela 1

Tendência das taxas de mortalidade infantil, neonatal e pós-neonatal. Porto Alegre, Rio Grande do Sul, Brasil, $1996-2008$.

\begin{tabular}{|c|c|c|c|c|}
\hline Ano de nascimento & $\begin{array}{l}\text { Número de } \\
\text { nascimentos }\end{array}$ & Mortalidade infantil & $\begin{array}{c}\text { Mortalidade } \\
\text { neonatal }\end{array}$ & $\begin{array}{l}\text { Mortalidade } \\
\text { pós-neonatal }\end{array}$ \\
\hline 1996 & 23.453 & 15,8 & 8,7 & 7,1 \\
\hline 1997 & 23.230 & 15,0 & 8,4 & 6,5 \\
\hline 1998 & 22.678 & 13,1 & 6,8 & 6,4 \\
\hline 1999 & 23.374 & 11,8 & 6,6 & 5,2 \\
\hline 2000 & 22.999 & 12,6 & 7,1 & 5,4 \\
\hline 2001 & 20.399 & 12,1 & 7,3 & 4,8 \\
\hline 2002 & 19.555 & 12,5 & 6,2 & 6,3 \\
\hline 2003 & 18.763 & 11,1 & 6,3 & 4,8 \\
\hline 2004 & 19.097 & 11,5 & 6,3 & 5,1 \\
\hline 2005 & 18.435 & 10,9 & 5,9 & 5,0 \\
\hline 2006 & 17.928 & 9,9 & 5,5 & 4,5 \\
\hline 2007 & 17.384 & 10,4 & 5,2 & 5,0 \\
\hline 2008 & 17.947 & 9,1 & 5,9 & 3,0 \\
\hline Total & 265.242 & 12,1 & 6,7 & 5,4 \\
\hline PMA & $-2,8$ * & $-3,7$ * & $-3,5$ * & $-4,1$ * \\
\hline IC95\% & $-3,4 ;-2,2$ & $-4,7 ;-2,8$ & $-4,7 ;-2,4$ & $-6,1 ;-2,2$ \\
\hline Valor de $p$ ** & & $<0,001$ & $<0,001$ & $<0,001$ \\
\hline
\end{tabular}

IC95\%: intervalo de 95\% de confiança; PMA: percentual de mudança anual.

* PMA significativamente diferente de zero;

** Teste qui-quadrado de tendência. 
Tendência de nascidos vivos e de mortalidade infantil de acordo com as variáveis estudadas. Porto Alegre, Rio Grande do Sul, Brasil, $1996-2008$.

\begin{tabular}{|c|c|c|c|c|c|c|c|c|c|c|c|c|}
\hline \multirow[t]{2}{*}{ Variáveis } & \multicolumn{6}{|c|}{ Taxa de nascidos vivos } & \multicolumn{6}{|c|}{ Taxa de mortalidade infantil } \\
\hline & $\mathrm{n}$ & 1996 & 2008 & PMA & IC95\% & $\begin{array}{l}\text { Valor } \\
\text { de } p \text { * }\end{array}$ & $\mathrm{n}$ & 1996 & 2008 & PMA & IC95\% & $\begin{array}{l}\text { Valor } \\
\text { de } p \text { * }\end{array}$ \\
\hline \multicolumn{13}{|c|}{ Escolaridade materna (anos) } \\
\hline$<8$ & 104.637 & 47,42 & 27,81 & $-4,5$ ** & $-5,6 ;-3,3$ & $<0,001$ & 1.774 & 20,10 & 13,84 & $-2,0 * \star$ & $-3,1 ;-0,9$ & 0,002 \\
\hline $8-11$ & 103.548 & 41,93 & 41,86 & $-0,3$ & $-1,9 ; 1,3$ & $<0,001$ & 1.055 & 12,39 & 8,00 & $-2,5^{\star *}$ & $-4,7 ;-0.3$ & 0,002 \\
\hline$\geq 12$ & 55.464 & 10,65 & 30,33 & 9,1 ** & 5,$7 ; 12,6$ & $<0,001$ & 335 & 7,26 & 5,89 & 0,6 & $-2,4 ; 3,6$ & 0,614 \\
\hline \multicolumn{13}{|c|}{ Consultas de pré-natal } \\
\hline Nenhuma & 12.551 & 7,47 & 2,69 & $-9,2$ ** & $-10,0 ;-8,3$ & $<0,001$ & 569 & 34,12 & 66,53 & 2,9 ** & 0,$4 ; 5,6$ & 0,011 \\
\hline $1-6$ & 89.092 & 33,43 & 26,78 & $-2,8$ ** & $-4,6 ;-1,1$ & $<0,001$ & 1.555 & 21,70 & 14,60 & $-0,6$ & $-31 ; 2,0$ & 0,488 \\
\hline$\geq 7$ & 161.925 & 59,11 & 70,54 & 2,3 ** & 1,$5 ; 3,1$ & $<0,001$ & 1.041 & 9,79 & 4,75 & $-4,7$ ** & $-6,8 ;-2,6$ & $<0,001$ \\
\hline \multicolumn{13}{|l|}{ Idade materna (anos) } \\
\hline$\leq 17$ & 24.008 & 9,48 & 7,42 & $-1,9$ ** & $-2,7 ;-1,2$ & $<0,001$ & 415 & 22,03 & 9,01 & $-4,5$ ** & $-6,9 ;-2,1$ & 0,001 \\
\hline $18-20$ & 38.411 & 14,56 & 12,93 & $-1,1$ ** & $-1,8 ;-0,4$ & $<0,001$ & 516 & 16,99 & 10,34 & $-2,9 * \star$ & $-5,3 ;-0,5$ & 0,010 \\
\hline $21-30$ & 124.427 & 47,30 & 48,15 & 0,3 ** & 0,$1 ; 0,6$ & $<0,001$ & 1.451 & 16,14 & 9,26 & $-3,6$ ** & $-5,1 ;-2,1$ & $<0,001$ \\
\hline $31-35$ & 46.940 & 18,19 & 18,73 & $-0,0$ & $-0,6 ; 0,6$ & 0,941 & 441 & 10,08 & 8,33 & $-4,5$ ** & $-6,9 ;-2,0$ & $<0,001$ \\
\hline$>35$ & 31.418 & 10,46 & 12,77 & $1,5^{\star \star}$ & 1,$0 ; 1,9$ & $<0,001$ & 395 & 16,72 & 8,29 & $-2,5$ & $-5,8 ; 1,0$ & 0,040 \\
\hline \multicolumn{13}{|l|}{ Filhos vivos } \\
\hline Nenhum & 117.985 & 43,39 & 48,04 & $1,4^{\star \star}$ & 1,$0 ; 1,8$ & $<0,001$ & 1.231 & 14,74 & 7,54 & $-4,2$ *夫 & $-5,7 ;-2,7$ & $<0,001$ \\
\hline $1-2$ & 108.940 & 41,44 & 40,50 & $-0,6$ ** & $-0,9 ;-0,3$ & $<0,001$ & 1.294 & 14,51 & 9,22 & $-3,3 * \star$ & $-4,5 ;-2,1$ & $<0,001$ \\
\hline $3-4$ & 27.241 & 10,94 & 8,52 & $-2,3$ ** & $-2,9 ;-1,6$ & $<0,001$ & 461 & 24,18 & 14,39 & $-4,8$ ** & $-7,0 ;-2,5$ & $<0,001$ \\
\hline$\geq 5$ & 10.633 & 4,23 & 2,94 & $-3,2$ ** & $-4,7 ;-1,7$ & $<0,001$ & 228 & 17,14 & 17,08 & 1,8 & $-3,3 ; 7,1$ & 0,435 \\
\hline \multicolumn{13}{|l|}{ Filhos mortos } \\
\hline Nenhum & 254.107 & 97,52 & 96,15 & $-0,3$ ** & $-0,5 ;-0,1$ & $<0,001$ & 3.006 & 15,53 & 8,93 & $-3,8 * *$ & $-4,8 ;-2,8$ & $<0,001$ \\
\hline$\geq 1$ & 10.587 & 2,48 & 3,85 & 4,5 & $-2,2 ; 11,8$ & $<0,001$ & 209 & 25,82 & 13,02 & $-6,2$ ** & $-10,2 ;-1,9$ & 0,001 \\
\hline \multicolumn{13}{|c|}{ Sexo do recém-nascido } \\
\hline Masculino & 135.872 & 51,17 & 50,40 & $-0,0$ & $-0,1 ; 0,1$ & 0,480 & 1.767 & 19,66 & 9,51 & $-5,0$ ** & $-6,4 ;-3,7$ & $<0,001$ \\
\hline Feminino & 129.360 & 48,83 & 49,60 & 0,0 & $-0,1 ; 0,1$ & 0,480 & 1.452 & 11,70 & 8,54 & $-2,3$ ** & $-4,0 ;-0,5$ & 0,001 \\
\hline \multicolumn{13}{|l|}{ Tipo de nascimento } \\
\hline Parto vaginal & 158.436 & 65,40 & 53,30 & $-2,1$ ** & $-2,4 ;-1,8$ & $<0,001$ & 1.910 & 13,95 & 8,89 & $-3,8 * \star$ & $-5,3 ;-2,2$ & $<0,001$ \\
\hline Parto operatório & 106.802 & 34,60 & 46,70 & 3,1 ** & 2,$7 ; 3,6$ & $<0,001$ & 1.311 & 19,22 & 9,31 & $-3,9$ ** & $-6,1 ;-1,6$ & $<0,001$ \\
\hline \multicolumn{13}{|l|}{$\begin{array}{l}\text { Idade gestacional } \\
\text { (semanas) }\end{array}$} \\
\hline$\leq 36$ & 22.717 & 7,15 & 9,85 & $2,7^{\star \star}$ & 1,$6 ; 3,8$ & $<0,001$ & 1.699 & 97,26 & 53,76 & $-5,0$ ** & $-6,3 ;-3,8$ & $<0,001$ \\
\hline $37-41$ & 238.525 & 90,44 & 89,77 & $-0,1$ & $-0,2 ; 0,0$ & $<0,001$ & 1.470 & 9,39 & 4,10 & $-5,1$ ** & $-6,8 ;-3,4$ & $<0,001$ \\
\hline$\geq 42$ & 3.721 & 2,41 & 0,38 & $-12,6$ ** & $-15,1 ;-10,0$ & $<0,001$ & 36 & 12,39 & 14,71 & $\star \star \star \star ~$ & $\star \star \star \star ~$ & 0,869 \\
\hline \multicolumn{13}{|l|}{ Peso ao nascer (g) } \\
\hline $500-999$ & 1.298 & 0,40 & 0,50 & $1,7^{\star \star}$ & 0,$0 ; 3,4$ & 0,035 & 825 & 744,68 & 573,03 & $-2,5 * \star$ & $-3,4 ;-1,6$ & $<0,001$ \\
\hline $1.000-1.499$ & 2.066 & 0,71 & 0,83 & 0,7 & $-0,6 ; 2,1$ & 0,206 & 365 & 283,13 & 114,09 & $-4,4$ ** & $-8,0 ;-0,7$ & 0,002 \\
\hline $1.500-2.499$ & 19.544 & 7,09 & 7,83 & 0,6 ** & 0,$2 ; 1,0$ & 0,002 & 619 & 41,49 & 21,34 & $-5,5$ ** & $-7,9 ;-3,0$ & $<0,001$ \\
\hline $2.500-2.999$ & 61.567 & 22,44 & 22,73 & 0,4 & $-0,1 ; 0,8$ & $<0,001$ & 568 & 11,97 & 6,62 & $-3,8$ ** & $-5,8 ;-1,7$ & 0,001 \\
\hline $3.000-3.999$ & 166.184 & 62,89 & 62,65 & $-0,1$ & $-0,2 ; 0,1$ & 0,090 & 779 & 7,39 & 3,20 & $-6,0 * \star$ & $-7,9 ;-4,1$ & $<0,001$ \\
\hline$\geq 4.000$ & 14.583 & 6,47 & 5,46 & $-1,8$ ** & $-2.8 ;-0.7$ & $<0,001$ & 65 & 7,91 & 2,04 & $-4,4$ & $-9,0 ; 0,3$ & 0,178 \\
\hline \multicolumn{13}{|l|}{ Tipo de hospital } \\
\hline Privado & 46.627 & 17,24 & 18,45 & $0,5^{* *}$ & 0,$2 ; 0,9$ & $<0,001$ & 209 & 9,67 & 2,73 & $-7,0$ ** & $-11,5 ;-2,4$ & $<0,001$ \\
\hline Misto & 87.882 & 31,72 & 32,61 & 0,0 & $-0,6 ; 0,7$ & 0,605 & 1.104 & 15,10 & 8,59 & $-3,7$ ** & $-5,5 ;-1,9$ & $<0,001$ \\
\hline Público & 129.904 & 51,05 & 48,94 & $-0,2$ & $-0,7 ; 0,2$ & $<0,001$ & 1.881 & 18,09 & 10,99 & $-3,5 * \star$ & $-4,9 ;-2,0$ & $<0,001$ \\
\hline
\end{tabular}

IC95\%: intervalo de 95\% de confiança; PMA: percentual de mudança anual.

* Teste qui-quadrado de tendência;

** PMA significativamente diferente de zero;

*** Não foi possível calcular o PMA nos estratos que não apresentaram óbito em um ou mais anos. 
anos e de 8-11 anos de escolaridade materna, 7 ou mais consultas de pré-natal, até 35 anos de idade materna, até 4 filhos vivos, até 41 semanas de idade gestacional e peso de nascimento até 3.999g. Houve tendência de aumento da mortalidade infantil entre os nascidos de mulheres que não realizaram o atendimento de pré-natal.

Na Tabela 3, nota-se que a redução da taxa de mortalidade infantil (TMI), para os nascidos de mães com menos de 8 anos de escolaridade, ocorreu em função da redução da taxa de mortalidade neonatal $(2,5 \%$ ao ano; valor de $\mathrm{p}=$ 0,007) e, para os nascidos de mães com 8-11 anos de escolaridade, em função da redução da taxa de mortalidade pós-neonatal (3,3\% ao ano; valor de $\mathrm{p}=0,005)$. Entre os nascidos de mães com 12 anos ou mais anos de escolaridade, não houve alterações significativas das TMI, tanto no período neonatal $(0,3 \%$ ao ano; valor de $\mathrm{p}=$ $0,989)$ como no pós-neonatal $(1,2 \%$ ao ano; valor de $\mathrm{p}=0,544$ ).

Na Tabela 4, observa-se que a TMI diminuiu $3,84 \%(1 / 0,963)$ ao ano. Quando ajustado para escolaridade materna, o percentual anual de redução decai para 1,94\%, indicando o efeito protetor dessa variável. $\mathrm{O}$ aumento da escolaridade materna foi o fator que apresentou o maior impacto para o declínio das taxas de mortalidade infantil, seguido das variáveis assistenciais e maternas. Por outro lado, quando ajustado para o grupo de variáveis do recém-nascido, o percen- tual anual de redução aumenta para $4,71 \%$, sugerindo uma contribuição negativa dessas variáveis para a redução das taxas de mortalidade ao longo do período. As variáveis ambientais pouco influenciaram essa tendência. Esse panorama foi semelhante para as taxas de mortalidade neonatal e pós-neonatal, com exceção das variáveis maternas, que, no período neonatal, pouco influenciaram na sua tendência de redução.

\section{Discussão}

Os achados mostram que Porto Alegre encontrase adiantada no fenômeno de transição demográfica e epidemiológica, com uma tendência de redução das TMI, neonatal e pós-neonatal e do número de nascidos vivos. Também são características desse processo a tendência de aumento da idade materna e a redução da multiparidade e dos nascimentos entre mulheres com baixa escolaridade.

Em relação à tendência da TMI entre as gestantes que não realizaram consultas de pré-natal, pode-se salientar que, ao mesmo tempo em que ocorre uma diminuição significativa do número de nascidos vivos nessa categoria, observa-se um aumento, inversamente proporcional, da taxa de mortalidade infantil. Esse fenômeno pode ser reflexo do processo de redução da pobreza em Porto Alegre, atingindo, em 2009, a menor taxa

Tabela 3

Tendência das taxas de mortalidade neonatal e pós-neonatal de acordo com os anos de escolaridade materna. Porto Alegre, Rio Grande do Sul, Brasil, 1996-2008.

\begin{tabular}{|c|c|c|c|c|c|c|c|}
\hline \multirow[t]{2}{*}{ Ano } & \multirow[t]{2}{*}{$\mathbf{n}$} & \multicolumn{3}{|c|}{ Mortalidade neonatal } & \multicolumn{3}{|c|}{ Mortalidade pós-neonatal } \\
\hline & & $<8$ & $8-11$ & $\geq 12$ & $<8$ & $8-11$ & $\geq 12$ \\
\hline 2001 & 20.235 & 8,7 & 6,8 & 4,7 & 7,6 & 3,5 & 0,9 \\
\hline 2002 & 19.411 & 6,6 & 7,3 & 4,4 & 10,6 & 4,3 & 2,4 \\
\hline 2003 & 18.644 & 8,9 & 5,0 & 4,6 & 8,0 & 3,7 & 1,7 \\
\hline 2004 & 19.043 & 7,0 & 6,9 & 4,4 & 7,3 & 4,8 & 2,8 \\
\hline 2005 & 18.376 & 8,3 & 4,9 & 4,7 & 7,9 & 4,5 & 2,3 \\
\hline 2006 & 17.855 & 7,3 & 6,1 & 2,5 & 7,7 & 3,6 & 2,5 \\
\hline 2007 & 17.367 & 5,4 & 5,6 & 4,6 & 11,2 & 2,9 & 2,0 \\
\hline 2008 & 17.924 & 8,2 & 5,9 & 3,5 & 5,4 & 2,0 & 2,0 \\
\hline Total & 263.649 & 8,3 & 6,3 & 4,0 & 8,6 & 3,9 & 2,1 \\
\hline PMA & & $-2,5$ * & $-2,0$ & $-0,3$ & $-1,5$ & $-3,3$ * & 1,2 \\
\hline IC95\% & & $-4,6 ;-0,3$ & $-4,9 ; 1,0$ & $-4,3 ; 4,0$ & $-4,2 ; 1,4$ & $-6,0 ;-0,5$ & $-3,1 ; 5,7$ \\
\hline Valor de $p$ ** & & 0,007 & 0,073 & 0,989 & 0,062 & 0,005 & 0,544 \\
\hline
\end{tabular}

IC95\%: intervalo de 95\% de confiança; PMA: percentual de mudança anual.

* PMA significativamente diferente de zero;

** Teste qui-quadrado de tendência. 
Risco relativo (RR) para mortalidade infantil, neonatal e pós-neonatal ajustadas pela escolaridade materna, variáveis assistenciais, maternas e do recémnascido. Porto Alegre, Rio Grande do Sul, Brasil, 1996-2008.

\begin{tabular}{|c|c|c|c|c|c|c|c|c|c|}
\hline \multirow{3}{*}{$\begin{array}{l}\text { Ano/Análise bruta e ajustada pelos conjuntos } \\
\text { de variáveis }\end{array}$} & \multicolumn{9}{|c|}{ Mortalidade } \\
\hline & \multicolumn{3}{|c|}{ Infantil } & \multicolumn{3}{|c|}{ Neonatal } & \multicolumn{3}{|c|}{ Pós-neonatal } \\
\hline & RR & IC95\% & Valor de p & RR & IC95\% & Valor de $p$ & RR & IC95\% & Valor de $p$ \\
\hline Ano & 0,963 & $\begin{array}{l}0,954 \\
0,972\end{array}$ & $<0,001$ & 0,965 & $\begin{array}{l}0,953 \\
0,977\end{array}$ & $<0,001$ & 0,957 & $\begin{array}{l}0,944 \\
0,971\end{array}$ & $<0,001$ \\
\hline Ano e escolaridade materna & 0,981 & $\begin{array}{l}0,971 \\
0,990\end{array}$ & $<0,001$ & 0,980 & $\begin{array}{l}0,968 \\
0,993\end{array}$ & 0,003 & 0,979 & $\begin{array}{l}0,965 \\
0,993\end{array}$ & 0,004 \\
\hline $\begin{array}{l}\text { Ano e variáveis assistenciais (número de consultas } \\
\text { de pré-natal; tipo de nascimento; tipo de hospital) }\end{array}$ & 0,979 & $\begin{array}{l}0,969 \\
0,989\end{array}$ & $<0,001$ & 0,981 & $\begin{array}{l}0,968 \\
0,994\end{array}$ & 0,005 & 0,974 & $\begin{array}{l}0,960 \\
0,989\end{array}$ & 0,001 \\
\hline $\begin{array}{l}\text { Ano e variáveis maternas (idade materna; número } \\
\text { de filhos vivos; número de filhos mortos) }\end{array}$ & 0,966 & $\begin{array}{l}0,957 \\
0,975\end{array}$ & $<0,001$ & 0,965 & $\begin{array}{l}0,952 \\
0,977\end{array}$ & $<0,001$ & 0,965 & $\begin{array}{l}0,951 \\
0,979\end{array}$ & $<0,001$ \\
\hline $\begin{array}{l}\text { Ano e variáveis do recém-nascido (sexo do recém- } \\
\text { nascido; idade gestacional; peso ao nascer) }\end{array}$ & 0,955 & $\begin{array}{l}0,946 \\
0,963\end{array}$ & $<0,001$ & 0,955 & $\begin{array}{l}0,944 \\
0,966\end{array}$ & $<0,001$ & 0,948 & $\begin{array}{l}0,934 \\
0,961\end{array}$ & $<0,001$ \\
\hline
\end{tabular}

IC95\%: intervalo de 95\% de confiança.

de pobreza entre as seis principais regiões metropolitanas do Brasil (regiões metropolitanas de São Paulo, Rio de Janeiro, Porto Alegre, Belo Horizonte, Salvador e Recife). Entretanto, concomitantemente à redução da pobreza, ocorreu um agravamento das condições materiais de vida entre os pobres. No período de 2005-2010, no conjunto das seis principais regiões metropolitanas do país, houve uma redução do desemprego $(-31,4 \%)$ acompanhada de um aumento nos dois decis com menor rendimento, sendo que, entre os trabalhadores pobres, a taxa de desemprego aumentou 28,5\% 29,30.

A redução da TMI e seus componentes entre os nascidos de mães com menos de 8 anos e com 8-11 anos de escolaridade, enquanto as taxas se mantiveram constantes entre os nascidos de mulheres com 12 ou mais anos de escolaridade, levou à redução das desigualdades na mortalidade infantil. Esses resultados são corroborados pelos achados de um estudo anterior, conduzido entre 1995 e 1999 na mesma cidade, no qual se verificou uma redução das TMI e da desigualdade nos estratos extremos à custa da redução das taxas de mortalidade neonatal e pós-neonatal 12 . A desigualdade no nível de renda possui um efeito contextual sobre as taxas de mortalidade. Wilkinson \& Pickett 31 , ao comparem as tendências de mortalidade entre cidades dos Estados Unidos, observaram que as cidades com maior igualdade de renda apresentavam uma redução nas taxas de mortalidade infantil em todas as categorias de renda, embora, paradoxalmente, permanecessem as desigualdades de acordo com o nível de renda.
Na população mais favorecida (com 12 ou mais anos de escolaridade), não houve alterações significativas nas taxas de mortalidade. Embora as taxas se encontrassem mais baixas do que nas demais categorias de escolaridade materna desde o início do período estudado, elas permanecem mais elevadas do que as taxas de mortalidade infantil de países desenvolvidos como a Islândia (2,9/1.000 nascidos vivos), o Japão $(3,2 / 1.000)$, a Suécia $(3,2 / 1.000)$ e a Noruega $(3,3 / 1.000) 32$. Para buscar compreender melhor esse fenômeno, compararam-se os nascidos vivos da categoria com doze ou mais anos de escolaridade com as demais categorias de escolaridade materna a partir das seguintes variáveis presentes no SINASC: idade materna, número de filhos vivos e mortos, sexo do recém-nascido, tipo de parto, idade gestacional, peso de nascimento e tipo de hospital. Assim, foi possível perceber que, nesse grupo, houve um percentual maior de partos operatórios $(67,6 \%)$ quando comparado com os nascidos vivos das categorias com escolaridade materna entre 8 e 11 anos $(40,5 \%)$ e com menos de 8 anos (25,7\%). A categoria mais favorecida apresentou também um pior prognóstico, com percentual mais elevado de nascimentos pré-termos $(63,3 \%)$ e percentual mais baixo de peso ao nascer entre 3.000 e $3.999 \mathrm{~g}$ $(18,2 \%)$, em comparação com as categorias de escolaridade materna entre 8 e 11 anos $(56,5 \%$ e $24,2 \%$, respectivamente) e com menos de 8 anos $(48,5 \%$ e $25,4 \%$, respectivamente). A realização indiscriminada de partos operatórios no grupo mais privilegiado, com consequente aumento 
de nascimentos pré-termo e de recém-nascidos com baixo peso ao nascer, pode ser responsável pela manutenção das taxas de mortalidade infantil ao longo do tempo.

Alguns estudos mostram que a redução da mortalidade infantil pode ser resultante de intervenções específicas das políticas públicas na área da saúde não acompanhadas de melhorias das condições socioeconômicas gerais da população 17,33,34. Entretanto, no presente estudo, a condição socioeconômica materna foi o fator com maior efeito positivo sobre a redução da mortalidade infantil, seguida dos fatores assistenciais e dos fatores resultantes da transição demográfica, presentes nas características maternas. Durante o período, ocorreu uma grande melhora da escolaridade materna em Porto Alegre 12 , e isso impactou as taxas de mortalidade infantil. Esse fenômeno é uma consequência do aumento dos anos de escolaridade das mulheres na região metropolitana de Porto Alegre, onde o percentual de mulheres com 8 ou mais anos de escolaridade aumentou de 46,09\% em 1996 para $60,98 \%$ em 2008, entre as mulheres com quinze ou mais anos de idade. Um considerável aumento na proporção de mulheres com 8 ou mais anos de escolaridade ocorreu também nas outras cinco principais regiões metropolitanas do país, sendo que a região metropolitana de Porto Alegre apresentou o segundo menor aumento (maior apenas que a do Rio de Janeiro), ficando, em 2008, com a segunda menor proporção de mulheres com mais de 8 anos de escolaridade, maior apenas que a de Recife (Departamento de Informática do SUS. http://www2.datasus. gov.br/DATASUS/index.php, acessado em 20/ Fev/2011). No Brasil, o aumento de acesso à assistência pré-natal também impactou positivamente as taxas de mortalidade 35 . Contudo, o aumento das taxas de muito baixo peso em Porto Alegre, no período do estudo, impactou negativamente a tendência de mortalidade. $\mathrm{O}$ aumento das taxas de natalidade entre mulheres acima de 40 e o acesso a novas tecnologias de reprodução são fatores que participaram desse fenômeno 8,36.

Neste estudo, são utilizadas informações sobre a escolaridade materna como forma de caracterizar as desigualdades de condições socioeconômicas e de acesso à saúde e suas repercussões sobre a mortalidade infantil, neonatal e pós-neonatal. Os efeitos da escolaridade sobre a saúde podem ser explicados de muitas maneiras como, por exemplo, a percepção dos problemas de saúde, a adoção de estilos de vida saudáveis, a utilização dos serviços de saúde e pelo fato de ser um proxy das condições materiais de vida, de ambiente e de trabalho dos indivíduos e de seus grupos sociais 37 . É conhecido que a utilização dos serviços de saúde resulta de uma multiplicidade de fatores, entre eles, a situação socioeconômica 38. A investigação dessas variáveis ocorreu pela sua confiabilidade e disponibilidade no SINASC ao longo do período analisado e pela sua capacidade de refletir essas desigualdades sociais. O elevado percentual de linkage denota a qualidade no preenchimento das variáveis nos sistemas de informação do Município de Porto Alegre. Contudo, uma elevada completude não significa, necessariamente, uma informação de qualidade, ou seja, que as variáveis tenham sido preenchidas corretamente.

O estudo apresenta, como limitação, o fato de a variável escolaridade materna ser capaz de explicar apenas parcialmente a desigualdade de condições socioeconômicas da população. Entretanto, a pobreza pode ser concebida não apenas como a falta ou a insuficiência de renda, mas como privação de capacidades básicas para alcançar certos níveis minimamente aceitáveis. A escolaridade é um dos vários elementos que aumentam a liberdade da pessoa para levar um tipo de vida ou outro ${ }^{39}$. Nesse sentido, o uso da escolaridade para caracterizar a condição socioeconômica torna-se mais adequado que o uso da renda. Além do mais, no Brasil, existe um gradiente positivo entre anos de estudo e renda familiar 37 . Outras limitações percebidas, por tratar-se de um estudo com dados secundários, são a ausência de informações sobre a renda e o fato de algumas variáveis apresentarem-se de forma categórica. As forças deste estudo são a completude do conjunto de dados de nascidos vivos e de óbito em Porto Alegre e a confiança na definição das variáveis.

Em conclusão, os resultados revelam uma tendência de redução da mortalidade infantil e de seus componentes neonatal e pós-neonatal. A redução da TMI e seus componentes entre os nascidos de mães com menos de 8 anos e com 8-11 anos de escolaridade, enquanto as taxas se mantiveram constantes entre os nascidos de mulheres com 12 ou mais anos de escolaridade, levou à redução das desigualdades na mortalidade infantil. A melhoria da condição socioeconômica materna foi o fator que mais se associou com a redução da mortalidade infantil, enquanto o aumento do baixo peso ao nascer e do muito baixo peso ao nascer desacelerou a tendência de redução da mortalidade infantil. 


\section{Resumo}

Objetiva-se determinar as tendências de mortalidade infantil e de seus fatores de risco em Porto Alegre. Este é um estudo baseado nas informações do registro de nascidos vivos e de óbitos infantis no período de 19962008. Foi analisada a tendência temporal das taxas de mortalidade infantil (TMI) e de seus componentes, de acordo com a escolaridade materna, o número de consultas de pré-natal, idade materna, número de filhos vivos e mortos, sexo do recém-nascido, o tipo de parto, a idade gestacional, o peso de nascimento e o tipo de hospital. Foi utilizada a regressão sequencial de Poisson para estimar a influência da condição socioeconômica e os demais fatores de risco. A TMI decaiu entre os nascidos de mães com escolaridade inferior a 11 anos. Entre os nascidos de mães com 12 ou mais anos de escolaridade, não houve alterações significativas. A condição socioeconômica materna foi o fator que mais se associou à redução da mortalidade infantil. Contudo, a tendência de redução não atingiu todo o potencial devido ao aumento do baixo peso ao nascer.

Mortalidade Infantil; Coeficiente de Mortalidade; Iniqüidade Social; Recém-Nascido de Baixo Peso

\section{Colaboradores}

A. R. Hernandez contribuiu na análise e interpretação dos resultados, na redação, revisão e aprovação da versão final do artigo. C. H. Silva e M. Z. Goldani contribuíram na concepção do projeto, na análise e interpretação dos resultados, revisão e aprovação da versão final do artigo. M. Agranonik e F. M. Quadros contribuíram na análise e interpretação dos resultados, revisão e aprovação da versão final do artigo.

\section{Agradecimentos}

Agradecimento especial para Juarez Cunha, Eugênio Lisboa e demais membros da Equipe de Informações em Saúde da Coordenação Geral de Vigilância em Saúde da Secretaria Municipal de Porto Alegre. Esta pesquisa foi financiada pelo Conselho Nacional de Desenvolvimento Científico e Tecnológico (CNPq).

\section{Referências}

1. Departamento de Análise de Situação em Saúde, Secretaria de Vigilância em Saúde, Ministério da Saúde. Saúde Brasil 2007: uma análise da situação de saúde. Brasília: Ministério da Saúde; 2007.

2. Menezes AMB, Victora CG, Barros FC, Albernaz E, Menezes FS, Jannke HA, et al. Mortalidade infantil em duas coortes de base populacional no Sul do Brasil: tendências e diferenciais. Cad Saúde Pública 1996; 12 Suppl 1:S79-86.

3. Costa MCN, Azi PA, Paim JS, Silva LMV. Mortalidade infantil e condições de vida: a reprodução das desigualdades sociais em saúde na década de 90. Cad Saúde Pública 2001; 17:555-67.

4. Barros FC, Victora CG. Maternal-child health in Pelotas, Rio Grande do Sul State, Brazil: major conclusions from comparisons of the 1982, 1993, and 2004 birth cohorts. Cad Saúde Pública 2008; 24 Suppl 3:S461-7.

5. Goldani MZ, Barbieri MA, Bettiol H, Barbieri MR, Tomkins A. Infant mortality rates according to socioeconomic status in a Brazilian city. Rev Saúde Pública 2001; 35:256-61.
6. World Health Organization. The incidence of low birth weight: a critical review of available information. World Health Stat Q 1980; 33:197-224.

7. Barbieri MA, Silva AA, Bettiol H, Gomes UA. Risk factors for the increasing trend in low birth weight among live births born by vaginal delivery, Brazil. Rev Saúde Pública 2000; 35:596-602.

8. Homrich da Silva C, Goldani MZ, Moura Silva AA, Agranonik M, Bettiol H, Barbieri MA, et al. The rise of multiple births in Brazil. Acta Paediatr 2008; 97:1019-23.

9. Silva AAM, Barbieri MA, Gomes UA, Bettiol H. Trends in low birth weight: a comparison of two birth cohorts separated by a 15-year interval in Ribeirao Preto, Brazil. Bull World Health Organ 1998; 76:73-84.

10. Silva AAM, Bettiol H, Barbieri MA, Ribeiro VS, Aragão VM, Brito LG, et al. Infant mortality and low birth weight in cities of Northeastern and Southeastern Brazil. Rev Saúde Pública 2003; 37:693-8. 
11. Barros FC, Victora CG, Barros AJ, Santos IS, Albernaz E, Matijasevich A, et al. The challenge of reducing neonatal mortality in middle-income countries: findings from three Brazilian birth cohorts in 1982, 1993, and 2004. Lancet 2005; 365:847-54.

12. Goldani MZ, Benatti R, Silva AA, Bettiol H, Correa JC, Tietzmann M, et al. Narrowing inequalities in infant mortality in Southern Brazil. Rev Saúde Pública 2002; 36:478-83.

13. Ventura RN, Oliveira EM, Silva EMK, Silva NN, Puccini RF. Condições de vida e mortalidade infantil no Município do Embu, São Paulo. Rev Paul Pediatr 2008; 26:251-7.

14. Bezerra Filho JG, Kerr LRFS, Miná DL, Barreto ML. Distribuição espacial da taxa de mortalidade infantil e principais determinantes no Ceará, Brasil, no período 2000-2002. Cad Saúde Pública 2007; 23:1173-85.

15. Andrade SM, Soares DA, Matsuo T, Souza RKT, Mathias TAF, Iwakura MLH, et al. Condições de vida e mortalidade infantil no Estado do Paraná, Brasil, 1997/2001. Cad Saúde Pública 2006; 22:181-9.

16. Guimarães MJB, Marques NM, Melo Filho DA, Szwarcwald CL. Condição de vida e mortalidade infantil: diferenciaisintra-urbanosno Recife, Pernambuco, Brasil. Cad Saúde Pública 2003; 19:1413-24.

17. Campos TP, Carvalho MS, Barcellos CC. Mortalidade infantil no Rio de Janeiro, Brasil: áreas de risco e trajetória dos pacientes até os serviços de saúde. Rev Panam Salud Pública 2000; 8:164-71.

18. Mello-Jorge MHP, Gotlieb SLD, Soboll MLMS, Almeida MF, Latorre MRDO. Avaliação do sistema de informação sobre nascidos vivos e o uso de seus dados em epidemiologia e estatísticas de saúde. Rev Saúde Pública 1993; 27 Suppl:1-46.

19. Companhia de Processamento de Dados. Observatório da cidade de Porto Alegre. http://www2. portoalegre.rs.gov.br/observatorio/ (acessado em 20/Fev/2011).

20. Sauve RS, Robertson C, Etches P, Byrne PJ, DayerZamora V. Before viability: a geographically based outcome study of infants weighing 500 grams or less at birth. Pediatrics 1998; 101 (3 Pt 1):438-45.

21. Lemons JA, Bauer CR, Oh W, Korones SB, Papile LA, Stoll BJ, et al. Very low birth weight outcomes of the National Institute of Child health and human development neonatal research network, January 1995 through December 1996. NICHD Neonatal Research Network. Pediatrics 2001; 107:E1.

22. Imaizumi Y. Infant mortality rates in single, twin and triplet births, and influencing factors in Japan, 1995-98. Paediatr Perinat Epidemiol 2001; 15:346-51.

23. Kiely JL. The epidemiology of perinatal mortality in multiple births. Bull N Y Acad Med 1990; 66: 618-37.

24. Papageorghiou AT, Bakoulas V, Sebire NJ, Nicolaides KH. Intrauterine growth in multiple pregnancies in relation to fetal number, chorionicity and gestational age. Ultrasound Obstet Gynecol 2008; 32:890-3.

25. Blickstein I. Normal and abnormal growth of multiples. Semin Neonatol 2002; 7:177-85.
26. Garite TJ, Clark RH, Elliott JP, Thorp JA. Twins and triplets: the effect of plurality and growth on neonatal outcome compared with singleton infants. Am J Obstet Gynecol 2004; 191:700-7.

27. Szwarcwald CL, Leal MC, Andrade CLT, Souza Jr. PRB. Estimação da mortalidade infantil no Brasil: o que dizem as informações sobre óbitos e nascimentos do Ministério da Saúde? Cad Saúde Pública 2002 ; 18:1725-36.

28. Shimakura SE, Carvalho MS, Aerts DRGC, Flores R. Distribuição espacial do risco: modelagem da mortalidade infantil em Porto Alegre, Rio Grande do Sul, Brasil. Cad Saúde Pública 2001; 17:1251-61.

29. Instituto de Pesquisa Econômica Aplicada. Comunicado da presidência ${ }^{\circ}$. 25: desigualdade e pobreza no Brasil metropolitano durante a crise internacional: primeiros resultados. http://www. ipea.gov.br/sites/000/2/comunicado_presiden cia/comunicado_da_presidencia_n25_2.pdf (acessado em 15/Dez/2010).

30. Instituto de Pesquisa Econômica Aplicada. Comunicado do Ipea $\mathrm{n}^{\circ}$. 76: desemprego e desigualdade no Brasil metropolitano. http://www. ipea.gov.br/portal/images/stories/PDFs/comu nicado/110210_comunicadoipea76.pdf> (acessado em 15/Dez/2010).

31. Wilkinson RG, Pickett KE. Income inequality and socioeconomic gradients in mortality. Am J Public Health 2008; 98:699-704.

32. Population Division, Department of Economic and Social Affairs, United Nations. World population prospects: the 2006 revision - highlights. New York: United Nations; 2007.

33. Victora CG, Grassi PR, Schmidt AM. Situação de saúde da criança em área da Região Sul do Brasil, 1980-1992: tendências temporais e distribuição espacial. Rev Saúde Pública 1994; 28:423-32.

34. Leal MC, Szwarcwald CL. Características da mortalidade neonatal no Estado do Rio de Janeiro na década de 80: uma visão espaço-temporal. Rev Saúde Pública 1997; 31:457-65.

35. Goldani MZ, Barbieri MA, Silva AA, Bettiol H. Trends in prenatal care use and low birthweight in southeast Brazil. Am J Public Health 2004; 94:1366-71.

36. Da Silva CH, Agranonik M, Da Silva AA, Bettiol H, Barbieri MA, Goldani MZ. Secular trend of very low birth weight rate in Porto Alegre, Southern Brazil. J Biosoc Sci 2010; 42:243-53.

37. Comissão Nacional sobre Determinantes Sociais da Saúde. As causas sociais das iniquidades em saúde no Brasil. Rio de Janeiro: Editora Fiocruz; 2008.

38. Travassos C, Martins M. Uma revisão sobre os conceitos de acesso e utilização de serviços de saúde. Cad Saúde Pública 2004; 20 Suppl 2:S190-8.

39. Sen AK. Desigualdade reexaminada. Rio de Janeiro: Editora Record; 2008.

Recebido em 09/Mar/2011

Versão final reapresentada em 01/Jul/2011

Aprovado em 19/Ago/2011 